\title{
New Progress in Clinical Diagnosis, Treatment and Laboratory Testing of Acute Respiratory Infections in Children
}

\author{
Huan Zhao ${ }^{1 *}$, Xiaoyu Jiang2*, Lingyu Lu ${ }^{3 \#, ~ Q i n g ~ L i n ~}{ }^{4 \#}$ \\ ${ }^{1}$ Inspection Department of Chongzuo Maternal and Child Health Care Hospital, Chongzuo, China \\ ${ }^{2}$ Inspection Department of Nanning Third People's Hospital, Nanning, China \\ ${ }^{3}$ Inspection Department of Nandan Hospital of Traditional Chinese Medicine, Nandan, China \\ ${ }^{4}$ Department of Infectious Diseases of Debao County People's Hospital, Debao, China \\ Email: "13907819585@163.com, "38669739@qq.com
}

How to cite this paper: Zhao, H., Jiang, X.Y., Lu, L.Y. and Lin, Q. (2022) New Progress in Clinical Diagnosis, Treatment and Laboratory Testing of Acute Respiratory Infections in Children. American Journal of Molecular Biology, 12, 1-9. https://doi.org/10.4236/ajmb.2022.121001

Received: December 30, 2021

Accepted: January 24, 2022

Published: January 27, 2022

Copyright $\odot 2022$ by author(s) and Scientific Research Publishing Inc. This work is licensed under the Creative Commons Attribution International License (CC BY 4.0).

http://creativecommons.org/licenses/by/4.0/

\begin{abstract}
Acute respiratory infection in children (ARTI) is the most common childhood infectious disease, and its pathogens include bacteria, fungi, viruses, chlamydia, mycoplasma and rickettsia. In recent years, with the continuous development of pathogen detection methods, the diagnosis and treatment of acute respiratory infections has received more and more attention from clinicians. The clinical diagnosis and treatment of acute respiratory infections in children and the research of laboratory detection methods have also been continuously developed. The manuscript presents a review of progress in the clinical diagnosis, treatment and laboratory testing of acute respiratory infections in children by collecting references.
\end{abstract}

\section{Keywords}

Children, Respiratory Infections, Pathogens, Laboratory Testing, Acute

\section{Introduction}

Acute respiratory tract infection in children is often referred to as the common cold in children. It is medically called acute nasopharyngitis. It is the most common acute upper respiratory tract infection caused by viral infections, most of which are self-limiting; and the incidence is relatively high, the number of patients is large, prone to multiple complications, and the economic burden is heavy [1] [2]. Common complications are mainly manifested as otitis media, si-

"Tied to the corresponding author 
nusitis, suppurative pharyngitis, bronchitis, and chronic respiratory diseases such as asthma and chronic bronchitis. Acute exacerbations can occur, obstructive sleep disordered breathing and deterioration can occur, and it can also cause bacterial or viral infection in children. Acute respiratory infections in children refer to acute infections above the throat, also known as upper respiratory tract infections, which are the most common infectious diseases in children. The disease mainly affects the nose, nasopharynx and pharynx. 90\% of children's colds are caused by viruses, and can also be caused by bacteria or mycoplasma infections. Infantile colds can be onset throughout the year, with more onsets in winter and spring. Infants and young children are susceptible to this disease due to the anatomy and immune characteristics of the upper respiratory tract. Therefore, there are more incidences in early childhood and relatively few school-age children [3] [4] [5]. In order to understand the clinical diagnosis and treatment characteristics of pediatric acute respiratory tract infection and the new progress of laboratory testing, the clinical diagnosis and treatment characteristics of acute respiratory infection in children in recent years and the new progress of laboratory detection are summarized as follows.

\section{Clinical Characteristics of Acute Respiratory Tract Infection in Children}

Acute respiratory infections in children have an incubation period, generally between 1 and 3 days. Enterovirus infection has the shortest incubation period, while adenovirus and respiratory syncytial virus infection have the longest incubation period [6] [7]. The onset of respiratory infections in children is relatively sudden. In most early cases, there is a burning sensation in the nasopharynx, followed by general malaise, muscle aches, nasal congestion, runny nose, and sneezing. In the case of the virus uncoating, symptoms can reach a peak within 48 hours; and acute rhinitis or pharyngitis caused by rhinovirus or coronavirus infection generally does not have fever or only low fever symptoms; symptoms such as eyelid swelling, conjunctival hyperemia, photophobia, tearing, or throat mucosal edema can be seen. Whether pharyngitis and tracheitis occur is generally related to personal constitution or virus infection. In the early stage of infection, the nasal secretions are mostly watery and clear nasal mucus. Later, as the infection deepens, the secretions will be mucinous or purulent. However, even the mucinous and purulent secretions may not indicate bacterial infection; although severe coughing is rare, it can last for about 2 weeks; the appearance of purulent secretions or the occurrence of severe symptoms of lower respiratory tract infection may indicate that the closed eye virus is combined with or secondary to pathogenic bacteria infection. Colds in children are usually more severe than adults, and often accompanied by symptoms of lower respiratory tract infection, but also accompanied by digestive system reactions such as vomiting, diarrhea and other symptoms. In the absence of complications, the disease is mostly self-limited, with a course of about one week, usually 4 - 10 days [8]. 


\section{Pathogenesis of Acute Respiratory Tract Infection in Children}

Acute respiratory infections in children with rhinovirus are mainly transmitted through contact caused by contaminating the nasopharyngeal secretions of cold patients; the transmission routes include: "hand-eye" and "hand-nose"; it can also be transmitted by droplets, but the probability of droplet spread is not as fast as the spread of flu [9] [10] [11] [12]. After rhinovirus infection, the replication concentration of rhinovirus reached its peak at 48 hours after rhinovirus infection, and the transmission period can last for 3 weeks. People who are susceptible are mostly related to their physical fitness such as nutritional health status, upper respiratory tract abnormalities such as tonsil enlargement, and whether they smoke or not. There is no correlation between acute respiratory infections and cold. The main cause of a cold in the cold season is related to the type of virus infection, and part of it is caused by the environment, such as gatherings of family members. The degree of infection can be affected by the host's physical condition. Nasopharyngeal allergic diseases, fatigue, depression, and menstrual cycles can all cause aggravation; the nasal cavity and eyes are the main gateways for rhinovirus to enter the body and the initial site of rhinovirus infection is the nasopharynx. After the rhinovirus enters the nasal cavity, the virus is adhered to the $M$ cell intercellular adhesion molecule-I (ICAM-1) receptor in the adenoid lymphoepithelial area, and then is carried to the posterior nasopharynx by the movable mucus cilia in the nasal cavity; the virus replicates rapidly in this area and spreads throughout the nasal cavity; due to the spread of the virus, nasal secretions and nasal epithelial cell biopsy can reveal that the secretion of related inflammatory mediators such as bradykinin, prostaglandin, IL-1 and IL-8 will increase, which is closely related to the occurrence of clinical symptoms; although the mechanism of histamine is still unclear, dripping histamine into the nasal cavity can cause symptoms of a cold, but antihistamine treatment is still uncertain; parasympathetic nerve blockers have a certain effect on the relief of cold symptoms, indicating that the neural reflex mechanism plays a certain role in the pathogenesis of colds. The production of immune reactions such as IgA and interferon is generally relatively short, coupled with the diversity of viral antigens and the drift, so the body will be repeatedly infected multiple times. The scope and virulence of virus infection are related to pathological changes; respiratory mucosa is hyperemia and edema, and leakage or exudation occurs; single cell group repair is faster, with little change and less damage [13]-[18]. The degree of cell proliferation and degeneration caused by different viruses is not the same; the cilia of the nasal mucosa can be destroyed for 2 - 10 weeks; when severely infected, both the nasopharyngeal tube and middle ear canal can be blocked and continue to cause severe infection.

\section{Disease Diagnosis of Acute Respiratory Infections in Children}

Acute respiratory infections in children need to be differentiated from other re- 
lated diseases [13] [14] [15] [16]. The most important points for distinguishing ARI from general influenza are: From an epidemiological point of view, influenza is a widespread disease. It is most common in winter and spring, but acute respiratory infections in children can occur throughout the year, mostly in winter, spring and autumn. From a clinical perspective, influenza has obvious symptoms of systemic poisoning, most of which are high fever of $39^{\circ} \mathrm{C}-40^{\circ} \mathrm{C}$, lasting for 3 - 5 days; severe body pain and headache are common; fatigue and weakness may appear in the early stage which can last for 2 to 3 weeks and accompanied by sore throat, nasal congestion, sneezing and other symptoms; commonly severe chest discomfort and coughing, etc., can often be complicated by bronchitis or pneumonia, and can even be life-threatening. The most typical catarrhal symptoms of influenza are granular changes in the mucosa of the soft palate and bronchitis. Respiratory tract infections in children mainly manifest as headache, fever, body pain, fatigue and weakness and other systemic symptoms which are relatively few and mild; there may be sore throat, nasal congestion and sneezing; some patients may have mild to moderate chest discomfort and coughing and complications are relatively rare. Differential diagnosis can be made through auxiliary examinations: such as X-ray examination; influenza is more prone to complications than acute respiratory infections in children; but it may not be complicated by bronchitis or pneumonia. The chest X-ray examination can help the diagnosis of clinical propensity. In terms of laboratory tests, it takes 3 to 10 days to determine the pathogen by collecting nasopharyngeal swabs, nasal secretions, and throat swabs for virus isolation and culture; it can be used for rapid detection of influenza pathogens, with a sensitivity of more than $70 \%$ and a specificity of more than $90 \%$, and it takes about 24 hours; differential diagnosis can be made by the determination of antibody titer serum antibodies in the acute and convalescent stages, and the inferior turbinate mucosal prints can also be collected for differential diagnosis; A large number of columnar epithelial cell degeneration can appear on the first day of influenza; Aniline blue inclusion bodies can appear in the cytoplasm or nucleus; However, acute respiratory infections are dominated by pus cells, and inclusion bodies are rarely seen.

Differential diagnosis of acute respiratory tract infection in children and other nasal diseases: a allergic rhinitis will have an allergic history, and the disease has seasonal characteristics such as pollen or nasal overflow, nasal congestion with pruritus and perennial sneezing and other symptoms, increased eosinophils found in nasal secretion can be diagnosed as allergic rhinitis. Vasomotor rhinitis has no history of allergies; it is mainly manifested by sneezing, runny nose and intermittent vascular filling of the nasal mucosa; dry air can aggravate the symptoms; it can be determined whether there is bacterial or viral infection based on the presence or absence of purulent tears, crusts, and medical history. Atrophic rhinitis mainly manifests as patency of the nasal cavity, thinning of the nasal mucosa propria, decreased blood vessels, formation of crusts, foul smell, and hypoosmia, which can be differentiated and diagnosed. Deviated nasal septum, nasal polyps and other diseases can be diagnosed by rhinoscopy. 
In addition, acute respiratory infections in children need to be differentiated from certain acute infectious diseases such as epidemic cerebrospinal meningitis, measles, encephalitis, typhoid fever, polio, upper respiratory tract inflammation in the prodromal period of HIV infection, and typhus. According to dynamic observations, relevant laboratory examinations and symptoms and medical history are identified.

\section{Laboratory Examination of Acute Respiratory Infection in Children}

The simplest method of laboratory examination for children with acute respiratory infection is routine blood examination. The total number of white blood cells is normal or low, and an increase in the percentage of lymphocytes may indicate a viral infection; when the total number of white blood cells increases, when the proportion of neutrophils increased and the nucleus shifted to the left, it indicates a bacterial infection. Viral infections can be determined by methods such as enzyme-linked immunosorbent assay, immunofluorescence, virus isolation and identification, and serological diagnosis; for bacterial infections, sputum or throat swabs or anal swabs can be collected for bacterial culture and drug sensitivity tests for identification and analysis of bacterial types and drug sensitivity, so as to provide therapeutic drug options for the clinic. Other auxiliary examinations can be performed by chest X-ray and CT to determine whether the infection is present or not [17] [18] [19] [20].

\section{Treatment Plan for Acute Respiratory Infections in Children}

The treatment of acute respiratory tract infection in children focuses on symptomatic treatment. The commonly used drugs are as follows [21]-[26]: Ipratropium bromide or ipratropium bromide spray can effectively relieve the symptoms of runny nose and sneezing, and the effect is better if it is used on the first day of the early stage of the disease. There are certain adverse reactions, which mainly manifest as bloody mucus in $15 \%-20 \%$ of patients. Pseudoephedrine mainly acts on alpha-adrenergic receptors in the respiratory mucosa which can improve nasal mucosal congestion, but has a poor effect on other peripheral blood vessel alpha-receptors and the heart, and can reduce nasal congestion and improve sleep; however, the use time should not be too long, 3 to 5 days is appropriate. Antihistamines such as chlorpheniramine maleate (Chlorpheniramine) are the first-generation antihistamines that are effective in reducing rhinorrhea and sneezing. Non-sedating antihistamines have no anticholinergic effect, so the effect is uncertain. Antipyretic analgesics are effective on patients with headache, fever and muscle aches. The most commonly used drug is acetaminophen (paracetamol), which cannot be used with the anti-HIV drug zivuddine. Repeated use of aspirin will increase the amount of virus excretion, but will not improve the symptoms, so it is not recommended. Antitussives are mainly used to reduce 
coughing and are generally not recommended; they can be used as appropriate only when severe coughing affects work and rest; dextromethorphan is more commonly used. In addition to the above treatment options, there are also reports of drugs or therapies used such as vitamin C, zinc gluconates, breathing heating and humidifying gas. It can also be treated with some antiviral drugs such as ribavirin (virazole) or related antiviral oral liquids, etc. In addition, preventive nursing is an important means to reduce children's acute respiratory tract infection and avoid contact with cold patients, especially pollutants. Studies have reported that maintaining good personal hygiene and using anti-virus wipes or paper towels can reduce the spread of rhinovirus. Vitamin $\mathrm{C}$ is advocated as a cold prevention drug, Vitamin $\mathrm{C}$ is recommended for use as a cold preventive agent, while Interferon $\alpha$-2b may be used after exposure to prevent the effects but may cause an adverse reaction to nasal congestion and discontinue use [27]-[32].

\section{Summary and Prospect}

Acute respiratory tract infection in children is mainly caused by respiratory pathogens. Atypical respiratory pathogens refer to pathogens other than Streptococcus pneumoniae, including Legionella pneumophila (LP), Mycoplasma pneumoniae (MP), Chlamydia pneumoniae (CP), Q Rickettsia fever (QFR), adenovirus (ADV), respiratory syncytial virus (RSV), influenza virus (IFV) and parainfluenza virus (PIV), etc. These pathogens have rapid variation and wide spread, and are not easy to form long-term immunity in the body, so it is difficult to prevent and control them. Bacteriological examination can be carried out by sputum culture or six joint tests of respiratory pathogens. Among various laboratory diagnostic methods, the antigen detection of respiratory virus by direct immunofluorescence method takes a short time. Generally, the results can be obtained two hours after receiving the sample. The requirements of laboratory conditions are not high, and it is simple, rapid, specific and sensitive; it is suitable for laboratory testing in primary medical institutions [33] [34] [35].

The incidence of acute respiratory tract infection in children is high, and the mortality rate is also relatively high. The main pathogen is viral infection, and the clinical manifestations include fever, cough, sore throat, fatigue, etc., without specific characteristics. At present, there are not many vaccines to prevent respiratory virus infection. In conclusion, understanding the clinical diagnosis and treatment characteristics and laboratory detection methods of acute respiratory tract infection in children has important clinical and practical significance for the treatment of respiratory tract infection in children.

\section{Acknowledgements}

In the research process of this subject, we have received strong support and help from many departments, individuals and other personnel who did not participate in the research of this subject. Here, all the members participating in this 
study express their deepest thanks to them and wish them good health and all the best.

\section{Fund Project}

Guangxi Hechi City Scientific Research and Technology Development Program (NO.: Heke Division 125504).

\section{Conflicts of Interest}

The authors declare no conflicts of interest regarding the publication of this paper.

\section{References}

[1] Wang, S.Y., Zhang, T.T., Zhang, X.M., et al. (2020) Study on the Epidemiological Characteristics and Economic Burden of Acute Respiratory Diseases in Children under 5 in Gansu. China Maternal and Child Health Care, 35, 2548-2552.

[2] Zhao, Y.Y. (2020) The Value of C-Reactive Protein and Blood Routine Testing in Children with Acute Upper Respiratory Tract Infection. Contemporary Medicine, 26, 140-142.

[3] Hu, S.Y. (2020) The Value of Combined Application of High-Sensitivity C-Reactive Protein and Blood Routine in the Diagnosis of Acute Upper Respiratory Tract Infection in Children. Great Doctor, 5, 46-48.

[4] Ma, H.L. and Ning, L.F. (2020) The Value of Serum C-Reactive Protein, B-Type Brain Natriuretic Peptide, Procalcitonin and Prealbumin in the Diagnosis and Efficacy Evaluation of Children with Acute Respiratory Infections. Journal of North Sichuan Medical College, 35, 52-56.

[5] Pan, H.Y., Lu, H.T. and Liu, C.J. (2020) The Diagnostic Value of Serum Procalcitonin and C-Reactive Protein in Children with Acute Upper Respiratory Tract Infection. Heilongjiang Medicine, 33, 690-692.

[6] Li, Z.Z. (2019) Analysis of Clinical Characteristics of Children with Acute Upper Respiratory Tract Infection in Nanning Area. Chinese Community Physician, 35, $150,153$.

[7] Wang, Z.X. (2019) Distribution Characteristics of Pathogenic Bacteria in Children with Acute Upper Respiratory Tract Infection and Treatment Recommendations. Clinical Medicine Research and Practice, 4, 73-74.

[8] Cheng, Y. (2019) The Use and Value Analysis of Antibacterial Drugs for Acute Upper Respiratory Tract Infection in Pediatric Outpatient Clinics. Lingnan Journal of Emergency Medicine, 24, 33-36.

[9] Ma, D.Q. and Wang, H. (2019) Etiological Investigation of Common Acute Respiratory Infections in Children in 2018. Chinese Community Physician, 35, 52, 54.

[10] Xiong, R.Z. (2019) Analysis of the Diagnosis and Treatment of Acute Respiratory Infections in Children with Bronchial Asthma. Chinese Continuing Medical Education, 11, 138-140.

[11] Wei, W.Y. (2019) Analysis of Respiratory Tract Etiology and Clinical Characteristics of 520 Cases of Acute Exacerbation of Bronchial Asthma in Children. China Modern Medicine Application, 13, 18-19.

[12] Ma, J.H. (2020) The Etiology and Clinical Treatment of Repeated Respiratory Infec- 
tions in Children. China Health Care and Nutrition, 30, 89.

[13] Zhu, L.Q. and Li, X.J. (2020) Application of Nucleic Acid Detection of Respiratory Pathogens in the Diagnosis of Acute Respiratory Diseases in Children. Shenzhen Journal of Integrated Traditional Chinese and Western Medicine, 30, 53-55.

[14] Shen, X.Y. and Zou, S.C. (2020) Epidemiological Analysis of Mycoplasma pneumonia Infection in Children with Respiratory Tract. Electronic Journal of Clinical Medicine, $7,173$.

[15] Han, L. and Wu, Y.L. (2020) Application Analysis of Serum PCT Combined with Blood Routine in the Differential Diagnosis of Acute Upper Respiratory Tract Infection in Children. Health Care Guide, No. 14, 273.

[16] Cai, X.X. (2020) The Application Value of Combined Detection of Serum PCT, hs-CRP, and Whole Blood WBC in Children with Acute Upper Respiratory Tract Infection. Modern Medicine and Health Research (Electronic Edition), 4, 146-147.

[17] Sun, C.Y. (2020) Detection and Analysis of Pathogens of Acute Upper Respiratory Tract Infection in Children. Digest of World Latest Medical Information (Continuous Electronic Journal), 20, 242-243.

[18] Xiao, J., Ying, J. and Li, M. (2019) The Distribution and Drug Resistance Analysis of Pathogenic Bacteria in Children with Acute Respiratory Infections and the Auxiliary Diagnosis Value of PTC and IL-6 Expression Levels. China Maternal and Child Health Care, 34, 4243-4245.

[19] Chen, X.J., Liu, Z.Q., Wu, X.C., et al. (2019) Distribution Characteristics of Pathogens in Acute Respiratory Infections in Children. Diet and Health Care, 6, 8-9.

[20] Tang, J. (2020) Application Analysis of Nine Joint Inspections of Respiratory Pathogens in Children with Acute Respiratory Diseases. World Latest Medical Information Abstracts (Continuous Electronic Journal), 20, 64-65.

[21] Miao, C.G. (2019) Analysis of the Rationality of Clinical Medication in 60 Children with Acute Upper Respiratory Tract Infection. Anti-Infective Pharmacy, 16, 1148-1150.

[22] Qian, H.C. (2020) Clinical Observation of Yinqiao Powder Combined with Western Medicine in the Treatment of Fever in Children with Acute Upper Respiratory Tract Infection. Electronic Journal of Integrated Traditional Chinese and Western Medicine Cardiovascular Diseases, 8, 182-186.

[23] Ma, Y.N., Hu, S.Y., Qiu, Y.P., et al. (2020) Meta-Analysis of Xiaoer Liaoqiao Qingre Granules versus Ribavirin in the Treatment of Acute Upper Respiratory Tract Infections in Children. Asia-Pacific Traditional Medicine, 16, 177-181.

[24] He, J.J. (2020) The Application Effect of Reducing Injection in the Treatment of Children with Acute Upper Respiratory Tract Infection with Fever. Wisdom Health, 6, 175-177.

[25] Lu, G.R. (2020) Explore the Clinical Effect of Pudilan Xiaoyan Oral Liquid in the Treatment of Acute Upper Respiratory Tract Infection in Children. Wisdom Health, 6, 185-186.

[26] Tang, C.M., Sui, H.H., Zhao, H.X., et al. (2020) Study on the Rationality of Clinical Medication for Acute Upper Respiratory Tract Infections in Children. Health Must Read, No. 9, 68-69.

[27] Wang, Y.Y. and Zhang, L.G. (2020) The Clinical Study of Xiaoer Jiegan Granules Combined with Cefuroxime axetil in the Treatment of Acute Upper Respiratory Tract Infections in Children. Modern Medicine and Clinics, 35, 1659-1662.

[28] Tao, B.Q. and Wang, J.Q. (2021) Efficacy and Safety of Jinlianhua Granule Combined with Antibiotics in the Treatment of Acute Respiratory Infections in Child- 
ren. Journal of Rare and Uncommon Diseases, 28, 21-22.

[29] Qian, Q.F. (2020) Comparison of the Effects of Azithromycin and Cefuroxime in the Treatment of Acute Bacterial Upper Respiratory Tract Infections in Children. Contemporary Medical Review, 18, 146-147.

[30] Li, H.B. and Liao, Y.Q. (2020) Study on the Effect of Comfort Nursing in the Nursing of Children with Acute Upper Respiratory Tract Infection. Special Health, No. 16, 186.

[31] Cui, J.M. (2020) Xiaoer Kechuanling Oral Liquid (Concentrated) Clinical Study on Treatment of Acute Upper Respiratory Tract Infection in Children. Jia You Yun Bao, 10, 17.

[32] Chen, L. (2020) Study on the Rationality of Clinical Medication for Acute Upper Respiratory Tract Infections in Children. Electronic Journal of Clinical Medicine Literature, 7, 15-16.

[33] Lu, Y.P., Cui, X.Y., Zhang, L., et al. (2020) Clinical Test Results of Mycoplasma bronchopneumoniae Infection in Children. Medical Dietetics and Health, 18, 187-188.

[34] Chen, J. and Yuan, C.Y. (2020) Analysis of Clinical Test Results of 40 Children with Mycoplasma pneumoniae Infection. Diet and Health Care, 7, 113-114.

[35] Lin, M.C., Li, X.J. and Li, D.F. (2020) Analysis of Detection Results of Seven Respiratory Viruses in Children. Chinese and Foreign Medical Care, 39, 177-179, 191. 